\title{
Simultaneous Demultiplexing of OTDM Channels Based on Swept-Pump Fiber-Optical Parametric Amplifier
}

\author{
Chi Zhang, Xie Wang, Xing Xu, P. C. Chui, and Kenneth K. Y. Wong* \\ The Photonic Systems Research Laboratory, Department of Electrical and Electronic Engineering, \\ The University of Hong Kong, Pokfulam Road, Hong Kong \\ kywong@eee.hku.hk
}

\begin{abstract}
We experimentally demonstrate simultaneous demultiplexing of $80-\mathrm{Gb} / \mathrm{s}$ OTDM signal by transforming it into WDM idlers (spaced by $1.15 \mathrm{~nm}$ ), based on a swept-pump fiber-optical parametric amplifier (FOPA), and $\sim 10$-dB parametric gain is achieved.

OCIS codes: (060.2320) Fiber optics amplifiers and oscillators; (060.4510) Optical communication; (190.4410) Nonlinear optics, parametric processes.
\end{abstract}

\section{Introduction}

With increasing demand for expanded transmission capacity, the optical time-division multiplexing (OTDM) and the wavelength-division multiplexing (WDM) techniques have been widely applied in current transmission systems $[1,2]$. The OTDM provides a simple solution for high-speed data generation beyond bandwidth limitation of the modulator, by time interleaving the low duty-cycle pulse trains at the same wavelength; however, the demultiplexing usually involved some complex sampling procedure or large bandwidth modulator [2,3]. While for the WDM system, different tributaries are combined with different optical carriers, and can be easily demultiplexed into separated channels by means of the arrayed waveguide gratings (AWG) filtering. Therefore, if we can first convert the OTDM signal into WDM signal, its demultiplexing procedure should be less demanding $[1,4,5]$. In this paper, we demonstrate a novel swept-pump fiber-optical parametric amplifier (FOPA) scheme, which is capable of converting the OTDM signal into WDM signal, and realizes the simultaneous OTDM demultiplexing.

\section{Principle}

In FOPA, two pump photons $\left(\omega_{p}\right)$ are annihilated to create signal $\left(\omega_{s}\right)$ and idler $\left(\omega_{i}\right)$ photons [6]. Consider a frequency-swept pump (middle plot in Fig. 1 (a)), if we have multi-pulses within time period, e.g. OTDM signals (bottom plot in Fig. 1 (a)), the newly generated idler pulses will be at different wavelengths because of the degenerate four-wave mixing (FWM) relation (top plot in Fig. 1 (a)) [7]. Since the repetition rate of idler is kept as that of the pump, this demultiplexer requires the pump repetition rate as high as $10 \mathrm{GHz}$. For 8 channels OTDM signal (top plot in Fig. 1 (b)), individual pulse interacts with different parts of the pump within one period, and 8channel idlers are generated with equally spaced optical carriers $\left(\lambda_{i 1}, \lambda_{i 2}, \ldots, \lambda_{i 8}\right)$; therefore demultiplexed in the time domain (Fig. 1(b)).

The performance of the FOPA demultiplexer is related to the quality of the generated ultrafast wideband sweptpump. One of the solutions is employing dispersive Fourier transformation (DFT) technique with a wideband short pulse. DFT is a technique that maps the spectrum of an optical pulse onto a time-domain waveform using groupvelocity dispersion (GVD) in a dispersive element (e.g. dispersive fiber), and removes the speed limitation introduced by conventional cavity configuration $[7,8]$. The wideband short pulse can be generated through some nonlinear effects, including the self-phase modulation (SPM), cross-phase modulation (XPM), and supercontinuum. The spectrum of the short pulse will be greatly expanded through these effects.

(a) $\uparrow 1$

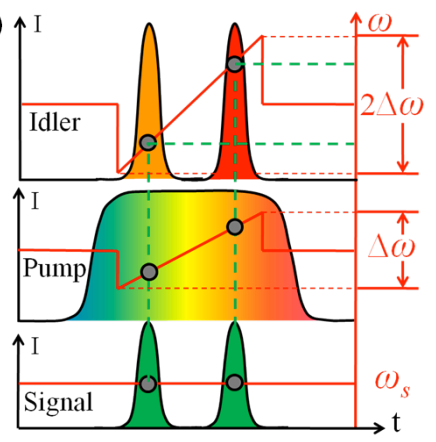

(b)

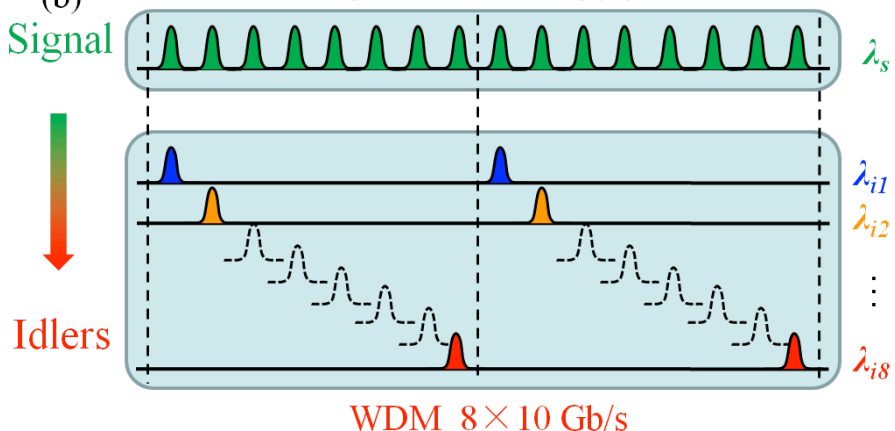

Fig. 1. Principle of the OTDM demultiplexer based on swept-pump FOPA: (a) Schematic diagram of the swept-pump FOPA, (b) Transformation from the OTDM signal to WDM idlers. 


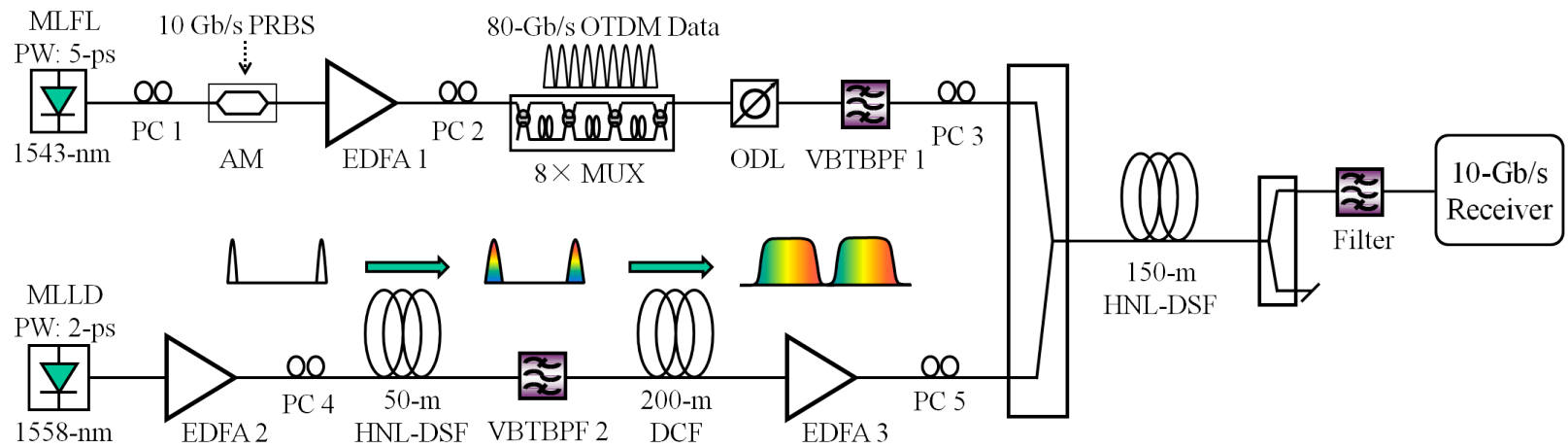

Fig. 2. Experimental setup for the OTDM demultiplexer based on swept-pump FOPA. PW: pulsewidth; PC: polarization controller; AM: amplitude modulator; EDFA: Erbium doped fiber amplifier.

\section{Experimental setup}

The experimental setup of the OTDM demultiplexing based on swept-pump FOPA is shown in Fig. 2. To generate 80-Gb/s OTDM return-to-zero (RZ) signal, a mode-locked fiber laser (MLFL) generated a 10-GHz 5-ps pulse train at the wavelength of $1543.3 \mathrm{~nm}$, and encoded by $10-\mathrm{Gb} / \mathrm{s} 2^{7}-1$ pseudorandom binary sequence (PRBS) pattern in an amplitude modulator (AM). The 10-Gb/s signal was multiplexed up to $80 \mathrm{~Gb} / \mathrm{s}$ by a bit-rate multiplier, and the EDFA 1 was used to compensate its loss. The variable bandwidth tunable bandpass filter (VBTBPF 1) before the FOPA filtered out the amplified spontaneous emission (ASE) noise introduced by EDFA 1, and provided an actively control of the signal pulsewidth, broadening the pulsewidth but ensuring no inter symbol interference (ISI). The 80$\mathrm{Gb} / \mathrm{s}$ OTDM signal trace is shown in the inset of the Fig. 4(a).

The bottom branch of the Fig. 2 shows the generation of the swept-pump. Another synchronized 10-GHz modelocked laser diode (MLLD) provided a 2-ps pulse train at the wavelength of $1557.5 \mathrm{~nm}$. The short pulses were then spectrally broadened by SPM in a 50-m highly-nonlinear dispersion-shifted fiber (HNL-DSF), with nonlinear coefficient of $14 \mathrm{~W}^{-1} \mathrm{~km}^{-1}$ and zero-dispersion wavelength (ZDW) at $1554.8 \mathrm{~nm}$. The VBTBPF 2 selected a relatively flat wavelength range between $1557.8 \mathrm{~nm}$ and $1561.5 \mathrm{~nm}$, and the filtered spectrum is shown in Fig. 3(a). These filtered wideband short pulses were coupled into a dispersive fiber, which was a $200-\mathrm{m}$ dispersion compensating fiber (DCF), and resulted in an ultrafast swept-source generated by the DFT process. This sweptsource was further amplified to $0.6 \mathrm{~W}$ by EDFA 3 , and combined with the OTDM signal; it then acted as the pump of FOPA. The optical delay line (ODL) was used to match the time slot between the swept-pump and the OTDM signal. Another spool of $150-\mathrm{m}$ HNL-DSF (with the same ZDW and nonlinear coefficient of $30 \mathrm{~W}^{-1} \mathrm{~km}^{-1}$ ) was employed as the gain medium of FOPA. As a result, the newly generated idler contains different tributaries in terms of the WDM system, and was selectively filtered out by a $0.8-\mathrm{nm}$ tunable bandpass filter.

\section{Results and discussion}

Figure 3 shows the performance of the DFT process in generating the swept-pump. Started from a wideband short pulse (2-ps), from 1557.8 to $1561.5 \mathrm{~nm}$, we can observe its spectrum before the DCF in Fig. 3(a). The dispersion and the accumulated group velocity delay of the 200-m DCF are shown in Fig. 3(b), since the bandwidth is $4 \mathrm{~nm}$, we can treat this dispersion line as quasi-linear. Figure 3(c) shows the temporal waveform of the dispersed swept-pump and it can be observed that, for each single time period, it looks similar to the reversed spectrum, the leading edge corresponding to the red-shifted wavelength, due to the negative dispersion (around $-22 \mathrm{ps} / \mathrm{nm}$ ).
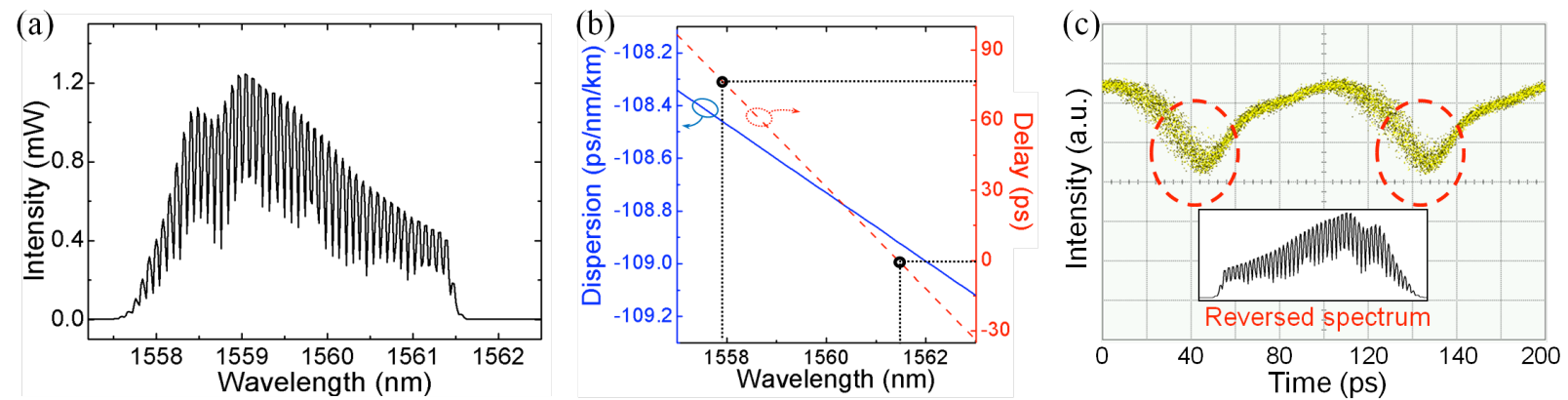

Fig. 3. Dispersive Fourier transformation (DFT) process: (a) Filtered pump spectrum before entering into the DCF; (b) Dispersion and accumulated group velocity delay of the $200-\mathrm{m} \mathrm{DCF}$; (c) Temporal waveform of the dispersed swept-pump, inset: reversed spectrum. 


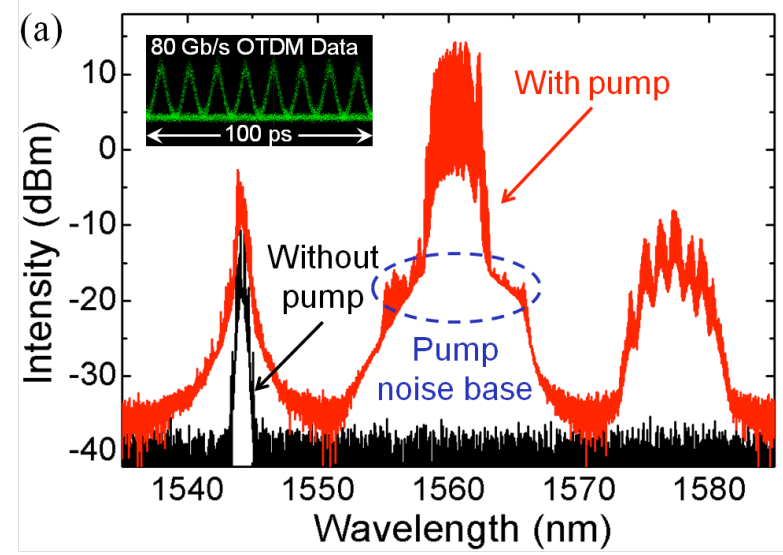

(c)

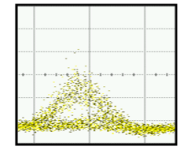

$1573.55 \mathrm{~nm}$

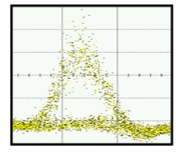

$1574.75 \mathrm{~nm}$

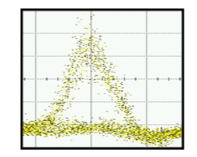

$1575.90 \mathrm{~nm}$
$1577.05 \mathrm{~nm}$
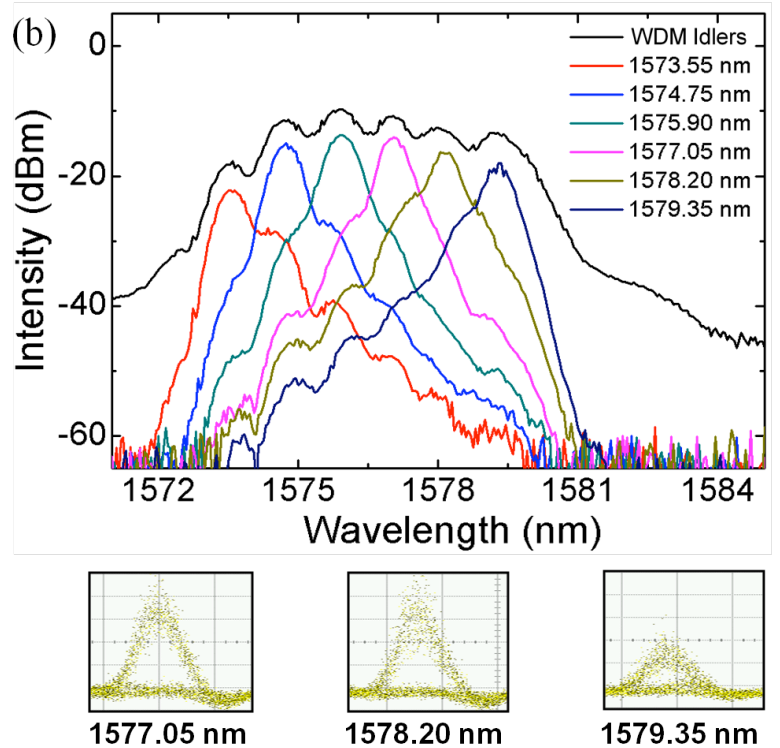

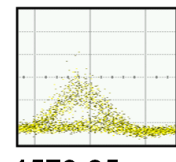

$1579.35 \mathrm{~nm}$

Fig. 4. OTDM demultiplexing results: (a) FOPA output spectra with or without the swept-pump, inset: $80 \mathrm{~Gb} / \mathrm{s}$ OTDM signal trace from the OSO; (b) Filtered spectra of WDM idlers, and corresponding wavelengths; (c) Eye-diagrams of each $10 \mathrm{~Gb} / \mathrm{s}$ WDM channels (without pre-amplifier).

The demultiplexing of the $80-\mathrm{Gb} / \mathrm{s}$ OTDM signal was achieved with swept-pump FOPA, the output spectra with/without the pump are shown in Fig. 4(a). At the signal side, the amplified power was $-1.2 \mathrm{dBm}$, and the on/off gain was measured to be $9.5 \mathrm{~dB}$, with the corresponding net gain of $6.5 \mathrm{~dB}$. At the idler side, as shown in Fig. 4(b), there were 6 channels observed, with the channel spacing of $1.15 \mathrm{~nm}$. Since the pump pulse shape and the filtered spectrum are not perfect square, which makes it hardly uniform to recover all the 8 channels simultaneously. If there was overlapping between two neighboring pulses, the falling edge (blue-shifted wavelength) of the front pulse would interfere the leading edge (red-shifted wavelength) of the later pulse. In the spectral domain, it can be observed that the FWM components arose at the two sides of the pump base (dash circle in Fig. 4(a)); while in the time domain, the interference was displayed as the intensity noise at the falling edge (dash circle in Fig. 3(c)). The eye-diagrams of each channels are shown in Fig. 4(c), clean eye opening is observed in the central four channels.

\section{Conclusion}

We have experimentally demonstrated an 80-Gb/s OTDM signal demultiplexing based on a swept-pump FOPA. The pump source was obtained by DFT technology, along with the SPM broadened spectrum. In the idler part, the newly generated $6 \mathrm{WDM}$ channels were spaced by $1.15 \mathrm{~nm}$, as well as $\sim 10-\mathrm{dB}$ parametric gain has been achieved. Since the swept-pump was not perfectly square, 4 channels have been demultiplexed with clean eye-diagrams. The 80 $\mathrm{Gb} / \mathrm{s}$ repetition rate could be further increased by employing dispersion-flattened fiber and larger swept-pump range.

\section{Acknowledgement}

The work described was partially supported by grants from the Research Grants Council of the Hong Kong Special Administrative Region, China (projects HKU 7179/08E and HKU 7183/09E). The authors acknowledge Sumitomo Electric Industries for providing the HNL-DSF and Alnair Laboratories for providing the VBTBPFs and MLLD.

\section{References}

[1] E. Palushani, H. C. H. Mulvad, M. Galili, H. Hu, L. K. Oxenlowe, A. T. Clausen, and P. Jeppesen, "OTDM-to-WDM Conversion Based on Time- to- Frequency Mapping by Time-Domain Optical Fourier Transformation," IEEE J. Sel. Top. Quantum. Electron., available online.

[2] Y. Liang, C. Zhang, and K. K. Y. Wong, "160-Gb/s Polarization-Insensitive Demultiplexer Based on a Fiber-Optical Parametric Amplifier," IEEE Photon. Technol. Lett., 23, $402-404$ (2011).

[3] K. Uchiyama, H. Takara, K. Mori, and T. Morioka, "160 Gbit/s all-optical time-division demultiplexing utilising modified multiple-output OTDM demultiplexer (MOXIC)," Electron. Lett., 38, 1190-1191 (2002).

[4] B. P. P. Kuo and S. Radic, "Fast wideband source tuning by extra-cavity parametric process," Opt. Exp., 19, 19930-19940 (2010).

[5] N. Satyan, G. Rakeljic, and A. Yariv, "Chirp multiplication by four wave mixing for wideband swept-frequency sources for high resolution imaging,” J. Lightw. Technol., 28, 2077-2083 (2010).

[6] M. E. Marhic, Fiber Optical Parametric Amplifiers, Oscillators and Related Devices (Cambridge University Press, 2007).

[7] C. Zhang, K. K. Y. Cheung, P. C. Chui, K. K. Tsia, and K. K. Y. Wong, "Fiber-Optical Parametric Amplifier With High-Speed Swept Pump," IEEE Photon. Technol. Lett., 23, 1022-1024 (2011).

[8] K. Goda, D. R. Solli, K. K. Tsia, and B. Jalali, "Theory of amplified dispersive Fourier transforamtion," Physical Review A, 80, 043821 (2009). 